\title{
BMJ open Female sex work interventions and changes in HIV and syphilis infection risks from 2003 to 2008 in India: a repeated cross-sectional study
}

\author{
Paul Arora, ${ }^{1}$ Nico J D Nagelkerke, ${ }^{2}$ Rahim Moineddin, ${ }^{3}$ Madhulekha Bhattacharya, ${ }^{4}$ \\ Prabhat Jha ${ }^{1}$
}

To cite: Arora $P$,

Nagelkerke NJD,

Moineddin $\mathrm{R}$, et al. Female sex work interventions and changes in HIV and syphilis infection risks from 2003 to 2008 in India: a repeated cross-sectional study. BMJ Open 2013;3:e002724. doi:10.1136/bmjopen-2013002724

- Prepublication history and additional material for this paper is available online. To view these files please visit the journal online

(http://dx.doi.org/10.1136/ bmjopen-2013-002724).

Received 13 February 2013

Revised 8 April 2013

Accepted 29 April 2013

This final article is available for use under the terms of the Creative Commons Attribution Non-Commercial 2.0 Licence; see http://bmjopen.bmj.com

For numbered affiliations see end of article.

Correspondence to

Paul Arora;

paul.arora@utoronto.ca

\section{ABSTRACT}

Objectives: We examined if increased spending and coverage of female sex worker (FSW) interventions were associated with declines in HIV or syphilis risk among young pregnant women (as a proxy for new infections in the general population) in the high-burden southern states of India.

Design: Repeated cross-sectional analysis.

Setting: We used logistic regression to relate district-level spending, number of sexually transmitted infections (STIS) treated, FSWs reached or condoms distributed to the declines in the annual risk of HIV and syphilis from 2003 to 2008 among prenatal clinic attendees in the four high-HIV burden states of Andhra Pradesh, Karnataka, Maharashtra and Tamil Nadu.

Participants: 386961 pregnant women aged 1524 years (as a proxy for incident infections in the adult population).

Interventions: We examined National AIDS Control Organisation (NACO) data on 868 FSW intervention projects implemented between 1995 and 2008.

Primary and secondary outcome measures: HIV or syphilis infection.

Results: HIV and syphilis prevalence declined substantially among young pregnant women. Each additional STI treated (per 1000 people) reduced the annual risk of HIV infection by $-1.7 \%(95 \% \mathrm{Cl}-3.3$ to -0.1$)$ and reduced the annual risk of syphilis infection by $-10.9 \%(95 \% \mathrm{Cl}-15.9$ to -5.8). Spending, FSWs reached or condoms distributed did not reduce HIV risk, but each was significantly associated with reduced annual risk of syphilis infection. There were no major differences between the NACO-funded and Avahan-funded districts in the annual risk of either STI. Conclusions: Targeted FSW interventions are associated with reductions in syphilis risk and STI treatment is associated with reduced HIV risk. Both more and less costly FSW interventions have comparable effectiveness.

\section{INTRODUCTION}

Less than $0.3 \%$ of Indian adults are infected with HIV, but owing to India's large population, they constitute a substantial fraction of the global HIV burden. In 2008-2009, an

\section{ARTICLE SUMMARY}

Article focus

- In about 2008-2009, an estimated two million adults were living with HIV in India. The four high-burden southern and western states of Andhra Pradesh, Karnataka, Maharashtra and Tamil Nadu account for over $60 \%$ of estimated HIV infections, although they have only $30 \%$ of India's adult population.

- Heterosexual sex, particularly between female sex workers (FSW) and their male clients, is the major source of new HIV infections in India. Sex work interventions, which have focused on reducing transmission between FSWs and their male clients, have expanded considerably in the highburden states. Among pregnant women attending government hospitals (as a proxy for general population of adults), HIV prevalence has declined substantially in all the four high-burden states from about 2000 onwards.

- Here, we examine if increased spending and coverage of FSW interventions were associated with declines in HIV or syphilis risk among young pregnant women (as a proxy for new infections in the general population) in the high-burden states of India.

Key messages

- HIV and syphilis prevalence declined substantially among young pregnant women. Each additional STI treated (per 1000 people) was associated with a reduction in annual risk of HIV infection by about $2 \%$ and a reduction in the annual risk of syphilis infection by about $11 \%$.

- Spending, FSWs reached or condoms distributed were not associated with a reduction in HIV risk, but each was significantly associated with a reduction in the annual risk of syphilis infection. There was no major difference in the association between interventions in the NACO (Government of India)-funded or Avahan (Gates Foundation)-funded districts and the annual risk of either HIV or syphilis.

- Targeted FSW interventions are associated with reduced syphilis risk, and STI treatment is associated with reduced HIV risk. More and less costly FSW interventions are comparable in effectiveness. 


\section{ARTICLE SUMMARY}

Strengths and limitations of this study

- Our study is the first to evaluate the association of sex work interventions and declines in HIV and syphilis risk among young pregnant women. Further strengths include a large annually repeated sample size and a study population, which was tested for HIV and syphilis for reasons unrelated to their risk from these infections.

- FSW interventions were not randomly assigned to districts. Moreover, FSW intervention spending or coverage may have had effects beyond the district in which they operate.

estimated 1.9 million (1.5-2.5\%) adults were living with HIV in India. ${ }^{1}{ }^{2}$ Heterosexual sex, particularly between female sex workers (FSWs) and their male clients, is the major source of new infections. ${ }^{3}$ The four southern and western states of Andhra Pradesh, Karnataka, Maharashtra and Tamil Nadu account for about $60 \%$ of the estimated HIV infections, although they have only $30 \%$ of India's adult population. Adults in these high-burden states report non-regular sexual partnerships more commonly than adults in other states of India, and the high-burden states account for half of the Indian males aged 1549 years who report using FSWs. ${ }^{4}$ India, like many other low-income or middle-income countries, relies on HIV testing of pregnant women to monitor changes in prevalence among the general population of adults. Among pregnant women attending government hospitals, HIV prevalence has declined substantially in all the four highburden states from about 2000 onwards. ${ }^{5} 6$

'Targeted' interventions for FSWs typically aim at primary HIV prevention and deliver treatment for sexually transmitted infections (STIs), condom provision, behaviour change communication, creation of a supportive environment using community involvement and participation (peer-based educators) and linkage to treatment and support services. ${ }^{7}$ FSW interventions have expanded considerably, mostly in the high-burden states: the Indian government's National AIDS Control Organisation (NACO) has spent about US\$420 million since 1999 and the Bill and Melinda Gates Foundation's Avahan programme spent an additional US\$250 million since $2004 .^{89}$

Earlier evaluations of FSW interventions, mostly focused on the Avahan programme, report increased condom use during last transactional sex ${ }^{10}$ changes in national HIV prevalence ${ }^{11}$ or in specific states. ${ }^{11-13}$ Here, we examine if increased spending and coverage of FSW interventions were associated with changes in HIV or syphilis risk in India's high-burden southern states. We further examine differences between the NACO-funded and Avahan-funded districts.

\section{DATA AND METHODS}

\section{Definitions of targeted interventions for FSWs}

A NACO-funded targeted intervention evaluation group $^{14}$ collected data on 868 targeted intervention projects implemented between 1995 and 2008 by governments, non-governmental organisations (NGOs) or external agencies in all 116 districts (small administrative areas each with about 2 million people) of the four high-burden states, including information on the duration, typology, geographical coverage, expenditures, staffing and services provided. These intervention data were checked against NACO's existing Computerized Management Information System. Study teams visited the states and, where relevant, NGOs to collect coverage and financial data using standardised data forms. Over $90 \%$ of the interventions involved FSWs, their clients or STI treatment.

Targeted interventions aim to ensure availability and acceptability of male lubricated latex condoms. ${ }^{8} 14$ Condoms are supplied free through peers, outreach workers or 'pick-up boxes' or via social marketing. Outreach workers attempt to address the risk of infection through peer counselling, counselling and by creating safe private environments for individual or group discussion about safer sex practices. $^{8}$

We defined spending as the reported annual project budget in Indian rupees (in current terms) and coverage as the numbers of FSWs reached, STIs treated and condoms distributed (both free and socially marketed). Syndromic treatment for STIs follows WHO/NACO guidelines. ${ }^{15}$ The choice of treatment follows a decision tree with presumptive antibiotic treatment and acyclovir where genital vesicles are seen. Avahan interventions were placed selectively and not randomly in selected districts with the highest HIV prevalence. The 30 districts in Karnataka were mutually exclusively funded by NACO or Avahan. ${ }^{12}$ However, districts in Andhra Pradesh, Maharashtra or Tamil Nadu could have both funders running programmes. For our analyses, districts were partitioned into those funded, in part or in whole, by Avahan $(n=70)$, or those funded exclusively by the Indian federal or state government $(n=46)$. Some external partners, such as the US Agency for International Development, funded FSW interventions in specific states, but these generally conformed to NACO standards and were classified as such. All intervention variables were aggregated at the district level as annual sums and converted to per capita measures using the district population in the Indian 2001 census.

\section{Serological HIV and syphilis outcomes among young pregnant women}

We related FSW intervention spending and coverage to individual unlinked, anonymous HIV and syphilis status among 386961 young women (aged 15-24 years) tested from 2003 to 2008 at 253 public prenatal clinics across all 116 districts of the high-burden states. The year 2003 was chosen as the starting year as this was the first year where surveillance coverage was complete in the highburden states. Trends in prevalent infections at ages 15-24 years, shortly after initiation of sexual activity and/or marriage,${ }^{16}$ are a reasonable proxy for trends in 
incident or new infections in the general population because prenatal clinic attendance is unrelated to HIV/ STI testing. In 2006, among women who had one live birth in the past 5 years, $89-99 \%$ of women in the four high-burden states reported at least one prenatal care visit. ${ }^{16}$ There was no significant change in the age, literacy status and urban/rural locality from 2003 to 2008 of women attending prenatal clinics (data not shown). HIV positivity was determined by two positive ELISA results, as per WHO guidelines. Syphilis positivity was determined by the Venereal Disease Research Laboratory slide tests from 2003 to 2007. Ancillary data included individual data on 27411 male STI clinic attendees aged 20-29 years from 43 sites in 40 districts and summary (not individual) data on 2405248 voluntary counselling and testing centre attendees from 112 districts (web table S1).

\section{Causal framework and statistical model}

We quantified a causal framework (figure 1) that assumes: (1) increased programme spending increases coverage defined specifically as the number of STIs treated, FSWs reached, and condoms distributed and (2) increased spending or coverage reduces the probability of acquiring HIV and syphilis infection among young women attending prenatal clinics.

Our statistical methods took into consideration that prevalence of HIV and syphilis in those women declined in almost all the study districts ${ }^{5}$ and that FSW interventions were not randomised, often beginning in the districts reporting the highest HIV prevalence. ${ }^{13}$ We used logistic regression to examine whether higher spending or coverage per capita was associated with greater declines (slopes) in the annual risk of HIV or syphilis infection. A random intercept and slope (for year) at the district level accounted for (additional) interdistrict variation. Spending or coverage variables were time-varying covariates with a 1-year lag (2-year lags yielded similar results; data not shown). Time was measured as a continuous variable. Surveillance data prior to 2003 were incomplete, precluding testing of a parallel trends assumption. However, we adjusted for baseline variation in the district-level HIV prevalence in 2003, possible regression to the mean (by including an interaction term between baseline prevalence and year), as well as the age, literacy, urban/rural locality and state of each woman tested. The effect of a one-unit increase in spending or coverage is presented as the relative difference in annual risk of HIV or syphilis from 2004 to 2008. Additional analyses considered if Avahan funded the districts. Model details and performance are provided in the web appendix. Alternative model specifications did not alter the results (web table S2). Analyses were conducted in SAS V.9.3 (Cary, North Carolina, USA).

\section{RESULTS}

HIV and syphilis prevalence in pregnant women aged 15-24 years declined significantly from 2003 to 2008 in the four high-burden states (relative decline of $36.4 \%$ and $68.8 \%$ for HIV and syphilis; table 1). Syphilis prevalence declines were about double the relative and absolute declines in HIV prevalence.

The cumulative number of FSW interventions grew steadily from 2000 onwards and spending rose sharply from 2004 onwards with the start of the Avahan programme. Figure 2 shows the approximate distribution of FSW interventions from 1998 to 2008, showing only about 150 interventions in 50 districts in the year 2000 . By 2008, 419 interventions in 106 districts were in place.

Annual reported spending, as well as the number of STIs treated, FSWs reached and condoms distributed increased in each state and for the NACO-funded or Avahan-funded districts (figure 3; web table S3). There was a strong and consistent relationship between spending and coverage. An average annual increase in programme spending of rupees 1 lakh (100 000 or about US $\$ 1700$ in 2013) from 2000 to 2008 was significantly associated with an average annual increase of 40 FSWs reached (95\% CI 30 to 48), 53 STIs treated (95\% CI 43 to 62 ) and 11000 condoms distributed (95\% CI 9400 to $12600)$.

The annual risk of infection declined significantly among young pregnant women from 2004 to 2008 for HIV and from 2004 to 2007 for syphilis (table 2). The annual risk of HIV infection was significantly associated

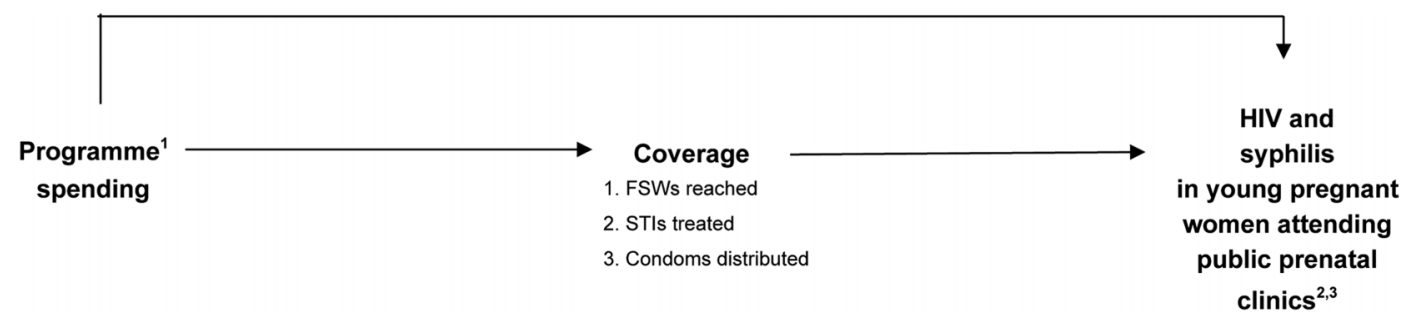

Figure 1 Causal framework for analysis of intervention impact. (1) Intervention data were aggregated at the district level and reported for each year. (2) HIV and syphilis data were available for pregnant women attending public prenatal clinics and male sexually transmitted infection (STI) clinic attendees; only HIV data were available for voluntary counselling and testing center (VCTC) attendees. (3) National AIDS Control Organisation HIV sentinel surveillance (NACO HSS) data were available as individual level data. 
Table 1 Age-specific prevalence of HIV and syphilis from 2003 to 2008 in high-burden states among women aged 15-24 years attending public prenatal clinics, overall and by state

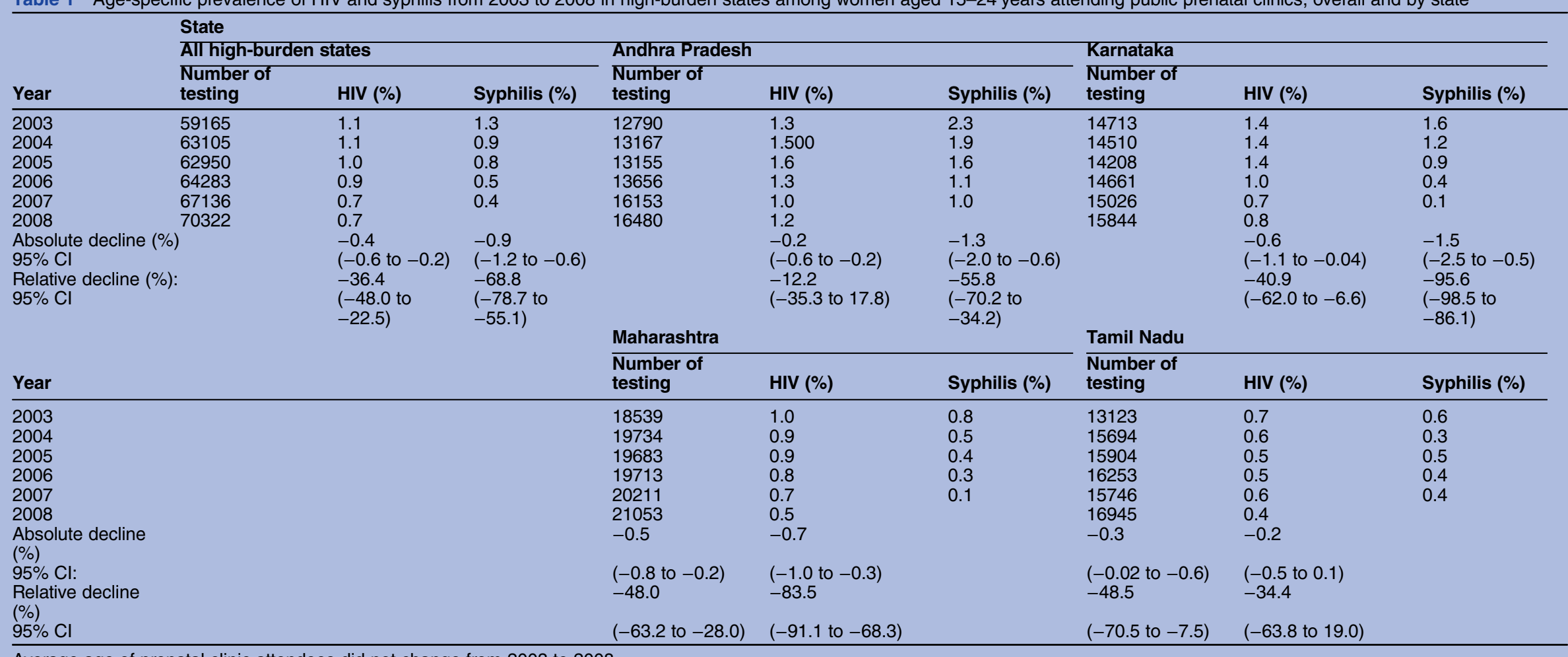

Average age of prenatal clinic attendees did not change from 2003 to 2008

Data from 116 districts of four high-burden states: Andhra Pradesh, Karnataka, Maharashtra and Tamil Nadu.

Number of sites per year: $2003=219,2004=234,2005=233,2006=235,2007=241$ and $2008=248$.

Syphilis data were only available until 2007. 

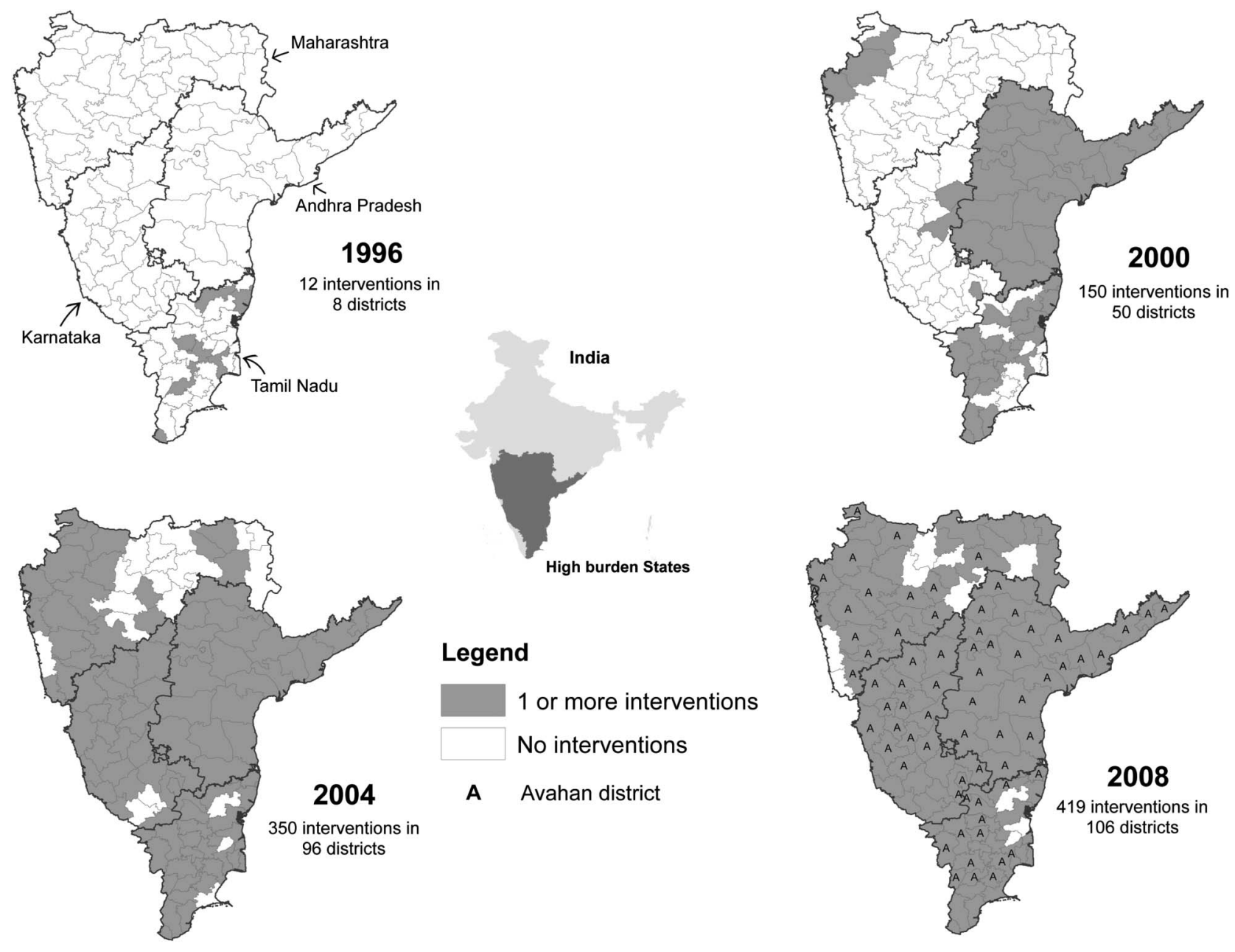

Figure 2 Growth in targeted interventions in South India. Avahan district markers are only shown in 2008; however, the Avahan programme began in 2004.

with STIs treatment: each additional STI treated per 1000 people in the previous year was associated with an additional $-1.7 \%$ (95\% CI -3.3 to -0.1$)$ decline in the annual risk of HIV infection. However, the decline in annual risk of HIV infection was not associated significantly with spending, FSWs reached or condoms distributed. The decline in annual risk of syphilis infection was significantly associated with spending, STIs treated, FSWs reached and condoms distributed, with the largest association observed with STIs treated. Each additional STI treated per 1000 people in the previous year was associated with an additional $-10.9 \%$ (95\% CI -15.9 to -5.8) decline in the annual risk of syphilis infection. The next largest association for syphilis risk arose for FSWs reached, condoms distributed and spending. Similarly, the largest association between intervention and HIV risk was with STIs treated. FSWs reached and condoms distributed were not significantly associated with HIV risk.

FSW intervention spending or coverage was associated with similar reductions in the annual risk of HIV infection across each of the four high-burden states (web table S4).
In Karnataka, spending and all coverage were associated with declines in syphilis infection. The largest reductions in annual risk of syphilis infection were associated with the number of STIs treated and FSWs reached.

The overall decline in annual risk of HIV or syphilis infection was similar in the NACO-funded or Avahan-funded districts. The annual risk of HIV infection was not significantly associated with spending or coverage in the Avahan-funded districts (table 3). By contrast, declines in the annual risk of HIV infection were significantly associated with spending or STIs treated in the NACO-funded districts; and these declines were statistically greater $(\mathrm{p}=0.01$ and 0.04 , respectively) than those observed in the Avahan-funded districts. STIs treated, FSWs reached and condoms distributed were all significantly associated with significant declines in the annual risk of syphilis infection in the Avahan-funded districts; however, the Avahan-NACO differences did not reach statistical significance. In both the Avahan-funded and NACO-funded districts, the largest reductions in annual risk of syphilis infection were associated with the number of STIs treated and FSWs reached. 

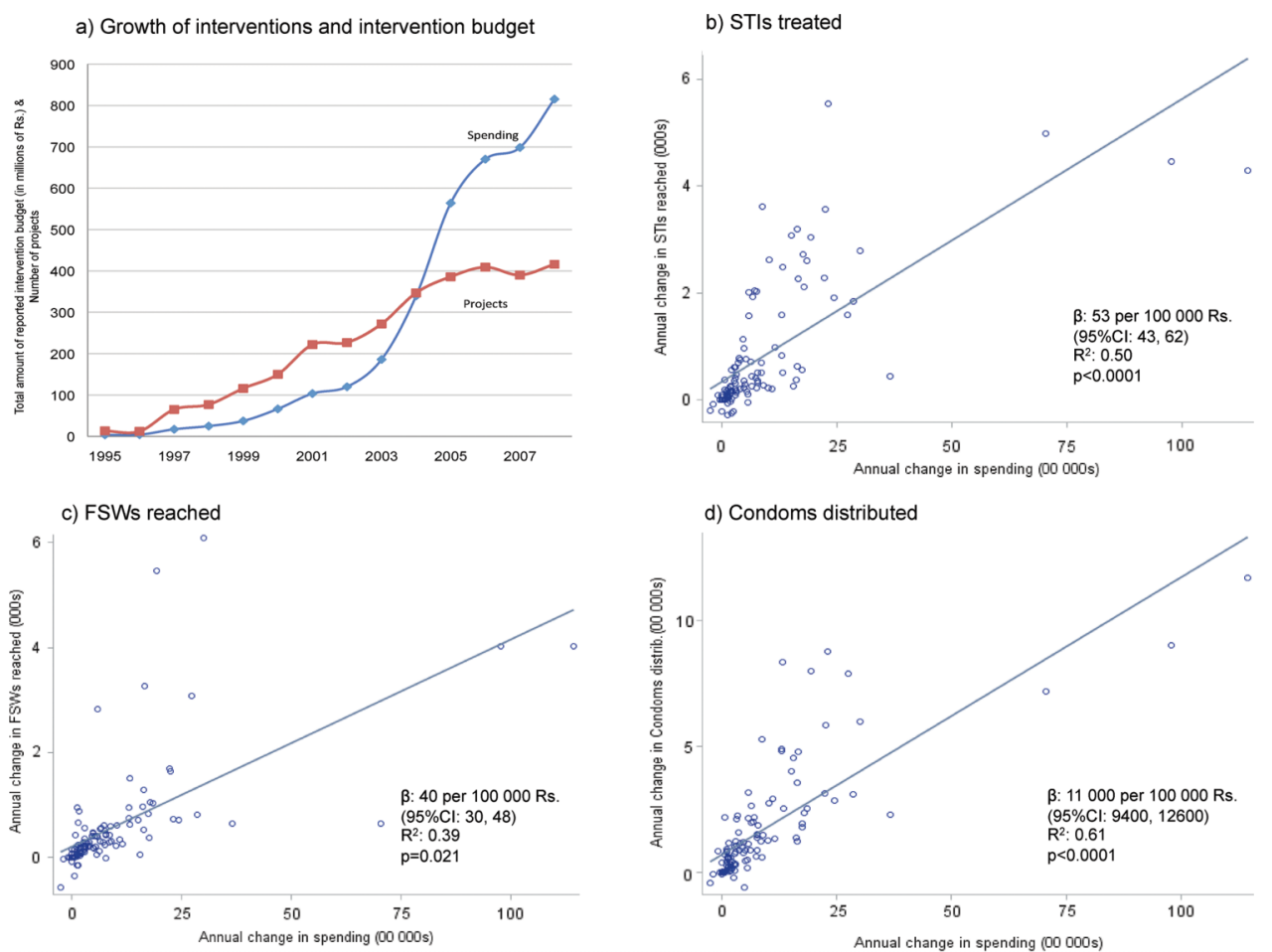

Figure 3 Association between change in intervention budget and change in intervention indicators from 2000 to 2008. (Rupees 100000 is about US\$1700 in 2013).

\section{DISCUSSION}

FSW intervention spending and coverage have substantially increased in India over the time period 2002-2008, with annual spending reaching about 800 million Indian rupees (about US\$13 million) funding interventions reaching about $50 \%$ of India's estimated 1.3 million FSWs. ${ }^{17}$ Among young pregnant women attending public prenatal clinics, STI treatment was associated with a reduction in the annual risk of HIV infection, which suggests that STI treatment reduced the incidence of HIV infections in the general population. However, we noted no significant association between greater spending or greater coverage with FSWs reached or condoms distributed and annual risk of HIV infection. By contrast, greater spending or coverage of FSW interventions were all significantly associated with reductions in the annual risk of syphilis infection in young pregnant women.

Treatment of STIs was associated with the largest reduction in the annual risk of both HIV and syphilis infection. Active STIs have frequently been shown to be a cofactor for HIV acquisition or transmission (particularly increasing the efficiency of male-to-female transmis$\operatorname{sion}^{18}$ ), and coexisting STIs thus seem integral in the transmission chain between male use of FSWs, male acquisition of HIV or syphilis and the subsequent spread to wives or regular female partners (figure 1). Among heterosexual HIV discordant partnerships in the Indian general population, the male partner introduces new HIV infections about $85 \%$ of the time, slightly more than for syphilis $(75 \%)$ or herpes simplex virus $2(64 \%)$, another common STI. ${ }^{19} 20$
There are several possible reasons why FSW intervention spending, numbers of FSWs reached or condoms distributed were not associated with a measurable reduction in the annual risk of HIV infection. First, we could evaluate lags of only 1-2 years due to a 6-year evaluation period. Thus, evaluation of the scale up of FSW interventions for the period 1995-2002 would be needed to estimate the association with more recent changes in HIV prevalence. However, regular testing of pregnant women was not practiced in all districts in the highburden states until 2003 onward. ${ }^{21}$ Second, HIV infection in India first appeared in 1986, and application of the Joint United Nations Programme on HIV/AIDS (UNAIDS) estimation and Project Package model ${ }^{22}$ suggests that its incidence in the adult general population might have peaked around 1996 at $0.06 \%$ and has been dropping steadily since (web figure S1). ${ }^{23}$ Thus, any effect of even a large increase in FSW interventions that began from 2003 onwards might be difficult to detect against the low and declining levels of new HIV infections in the general population. While less is known about trends in syphilis infection, it remains common in the high-risk populations in India ${ }^{24}$ (web table S1) and might respond more quickly to changes in spending or coverage. While transmissibility per sex act is about $3-20$ times greater for syphilis and other STIs than it is for $\mathrm{HIV}^{25}$ its duration of infectiousness is typically shorter and is further reduced by antibiotic treatments, which may explain why the incidence of syphilis infections might be more sensitive to disruptions in the transmission chain. This may be the reason why we observed a 
Table 2 Estimated impact of FSW interventions (spending or coverage) on annual risk of HIV or syphilis infection among pregnant women attending public prenatal clinics aged 15-24 years in four high-burden states from 2004 to 2008

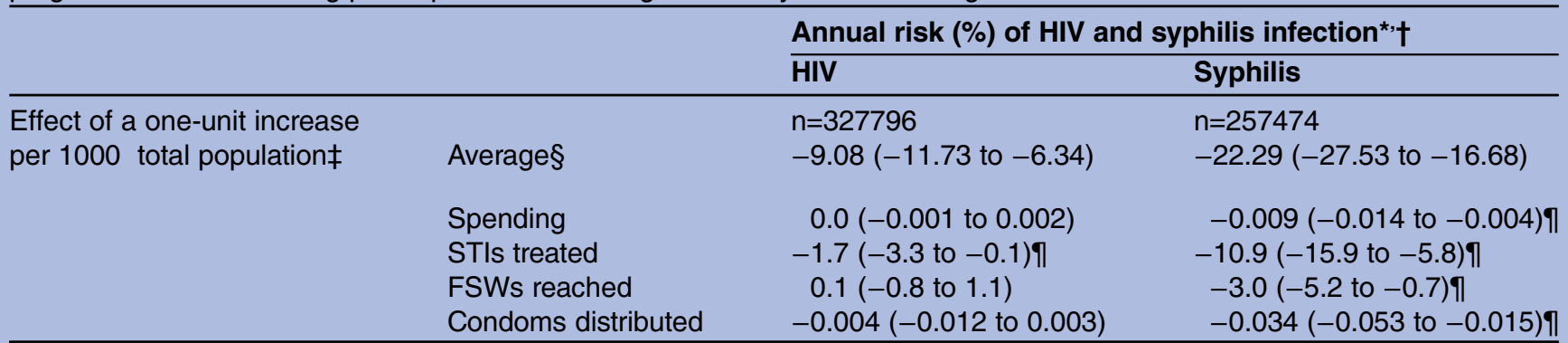

*Annual risk of infection is the OR for a one-unit increase in a 'year' expressed as percentage $=-(1-0 R) \times 100 \%$.

†Results are for the four high-burden states combined. Syphilis results were only available for $2007 . \mathrm{n}=$ number of pregnant women testing from 2004 to 2007/2008.

fEffect of spending or coverage is expressed as change (\%) in annual risk for a one-unit increase. All intervention effects are shown as a 1 -year lag.

$\S$ Average annual decline in the risk of HIV or syphilis infection from 2003 to 2007/2008.

TEffect of intervention with $p<0.05$.

FSW, female sex worker; STIs, sexually transmitted infections.

significant effect of STI treatment on reducing HIV risk. A small community-led HIV intervention to reduce sexual risk among $425 \mathrm{FSWs}$ in the city of Mysore, Karnataka noted large declines from 2004 to 2006 in syphilis, Trichomonas, chlamydia and gonorrhoea but not in HIV after 30 months of follow-up. ${ }^{26}$ Third, there might have been subtle differences at the state level in the sexual network structure or phase of the epidemic that could have prevented us from detecting an effect, ${ }^{13}$ even though our overall analyses controlled for statelevel differences. Karnataka, in particular, showed the strongest effects of FSW intervention spending and coverage (web table $\mathrm{S} 4$ ), consistent with a recent analysis of the Avahan-funded FSW interventions in that state. ${ }^{12}$

Our findings suggest that syndromic STI treatment in FSWs and their clients reduces HIV risk in the general population. By contrast, eight of the nine randomised controlled trials in African countries failed to

Table 3 Estimated impact of FSW interventions (spending or coverage) on annual risk of HIV or syphilis infection among pregnant women attending public prenatal clinics aged 15-24 years in four high-burden states from 2004 to 2008, in an Avahan-funded or NACO-funded district

\begin{tabular}{|c|c|c|c|c|}
\hline & & \multicolumn{2}{|c|}{ Annual risk of HIV or syphilis infection* } & \\
\hline & & NACO district & Avahan district $†$ & \\
\hline & $\begin{array}{l}\text { Intervention } \ddagger \\
\text { Average§ }\end{array}$ & $\begin{array}{l}\text { HIV } \\
n=141589 \\
-11.37(-15.99 \text { to }-6.51)\end{array}$ & $\begin{array}{l}\mathrm{n}=245372 \\
\quad-7.7(-11.04 \text { to }-4.25)\end{array}$ & $\begin{array}{l}\text { pdiff } \\
0.23\end{array}$ \\
\hline $\begin{array}{l}\text { Effect of a one-unit increase } \\
\text { per } 1000 \text { total population }\end{array}$ & $\begin{array}{l}\text { Spending } \\
\text { STIs treated } \\
\text { FSWs reached } \\
\text { Condoms distributed }\end{array}$ & $\begin{array}{l}-0.011(-0.019 \text { to }-0.003) \\
-5.84(-10.29 \text { to }-1.09) \\
-4.6(-9.74 \text { to } 0.8) \\
-0.025(-0.054 \text { to } 0.004) \\
\text { Syphilis } \\
n=117189\end{array}$ & $\begin{aligned} & 0.0(-0.001 \text { to } 0.002) \\
&-0.6(-2.57 \text { to } 1.31) \\
& 0.3(-0.7 \text { to } 1.21) \\
&-0.002(-0.01 \text { to } 0.006) \\
& n=199450\end{aligned}$ & $\begin{array}{l}0.01 \\
0.04 \\
0.08 \\
0.13\end{array}$ \\
\hline & Average§ & $-22.32(-31.33$ to -12.13$)$ & $-22.28(-28.7$ to -15.29$)$ & 0.99 \\
\hline $\begin{array}{l}\text { Effect of a one-unit increase } \\
\text { per } 1000 \text { total population } \emptyset\end{array}$ & $\begin{array}{l}\text { Spending } \\
\text { STIs treated } \\
\text { FSWs reached } \\
\text { Condoms distributed }\end{array}$ & $\begin{array}{c}-0.022(-0.035 \text { to }-0.01) \\
-12.0(-19.45 \text { to }-3.83) \\
-0.4(-10.29 \text { to } 10.68) \\
-0.036(-0.085 \text { to } 0.013)\end{array}$ & $\begin{array}{l}-0.007(-0.012 \text { to }-0.002) \\
-10.38(-16.01 \text { to }-4.41) \\
-3.25(-5.56 \text { to }-0.8) \\
-0.027(-0.049 \text { to }-0.006)\end{array}$ & $\begin{array}{l}0.03 \\
0.73 \\
0.61 \\
0.75\end{array}$ \\
\hline
\end{tabular}

*Annual risk of infection is the OR for a one-unit increase in a 'year' expressed as percentage $=-(1-O R) \times 100 \%$. Syphilis results were only available for 2007.

†70 districts (of 116) were Avahan districts from about 2004 onwards, $n=n u m b e r$ of pregnant women tested from 2003 to 2008 (or 2007 in the case of syphilis) and pdiff=test for difference in change in risk of infection between Avahan versus not districts.

$\ddagger$ All intervention effects are shown as a 1-year lag.

$\S$ Average annual decline in the risk of HIV or syphilis infection from 2003 to 2007/2008.

iEffect of intervention is expressed as change $(\%)$ in annual risk for a one-unit increase in intervention. Significant effects $(p<0.05)$ of interventions are indicated with bold text.

FSW, female sex worker; NACO, National AIDS Control Organisation; STIs, sexually transmitted infections. 
demonstrate that provision of STI treatment, or prevention of STI infection, significantly reduces HIV incidence. ${ }^{27-32}$ In each trial, however, there were significant modifying factors that made it difficult to draw firm conclusions about the effectiveness of STI treatment on reducing HIV incidence. ${ }^{18}$ Our analysis of STI treatment of high-risk groups for HIV prevention in the general population may have found an effect owing to the much longer duration of observation than in the African trials. Moreover, each of the nine randomised trials of STI treatment or prevention was powered to detect large reductions in HIV incidence (approximately 50\% reduction). It is possible that the effects of STI treatment are small, as observed in our study.

The Avahan-funded FSW interventions cost about twice as much as of the NACO-funded interventions. ${ }^{33}$ However, we find few differences in the association between interventions and the annual risk of HIV or syphilis infection in the NACO-funded or Avahan-funded districts. We were unable to assess the effects of specific interventions or activities, thus limiting our ability to compare the methods of delivery. Earlier analyses of Avahan FSW interventions noted that the changes in HIV prevalence did not significantly depend on the presence of Avahan interventions, ${ }^{11}$ and the magnitude and speed of the decline in HIV infection were larger than expected and most likely not solely attributable to the Avahan programme. ${ }^{34}$

FSW interventions were not randomly assigned to districts. Our analyses used a difference in declines approach, controlling for individual-level variables and for relevant district-level factors, most importantly the baseline prevalence and regression to the mean. This approach assumes parallel trends in outcome in districts with high versus low levels of interventions. We were not able to assess whether trends in HIV or syphilis were parallel prior to 2003 as HIV surveillance in the high-burden states was complete from 2003 and syphilis data were only available from 2002. However, a wide range of alternative statistical models did not materially alter our results (web table S2). Women attending public prenatal clinics are not representative of the general population. ${ }^{9}$ However, the large declines observed in HIV and syphilis in this population are not reasonably explained by any plausible change in characteristics of women attending antenatal clinics, expansion of testing sites, increases in female sterilisation, or expanded access to prenatal care. ${ }^{5}$ Moreover, in India, the time gap between age at first sex and age at first birth among women is small (about 2 years in the high-burden states), ${ }^{16}$ avoiding a possible bias noted in African countries. ${ }^{5}$ In India and other low HIV-prevalence settings, improvements in the specificity of HIV ELISA testing over time may falsely create declines in surveillance data. Annual false positivity rates in HIV testing were not available; however, NACO has adopted quality control measures for HIV testing, including repeat testing at national reference laboratories, and has used the same ELISA assays over the study period. ${ }^{35}$ Furthermore, improvements in testing quality should not have been differential between areas of high and low FSW intervention spending and high or low coverage. Finally, declines in HIV prevalence may also be driven by increasing AIDS mortality, but unlikely at ages 15 to 24 years and there was no notable change in the steady decline in all-cause death rates at these ages in the highburden states from 2003 to $2008 .^{36}$ Moreover, while India has worked to scale up access to antiretroviral therapy (ART) since its introduction in 2004, by 2008 less than 185000 people were receiving ART. ${ }^{37}$

In the high-burden states of India, the findings from sexual behaviour surveys in the general population and integrated biological and behavioural surveys among FSWs and their clients suggest that behaviours changed during the study period. From 2001 to 2009, among men of reproductive age in the south, self-reported condom use during the last sex act with a non-regular partner increased in all states except Maharashtra where it was already reported to be high. ${ }^{14}$ Similarly, FSWs reporting consistent condom use in the previous month increased from approximately $50-70 \%$ in 2001 to $75-$ $95 \%$ in $2009 .{ }^{14}$ Surveys among FSWs in these states from 2004 to 2009 suggest that the prevalence of STIs (syphilis, gonorrhoea and chlamydia) declined. ${ }^{10}{ }^{38-40}$ The integration of rapid diagnostic technologies into STI surveillance in high-risk groups may improve the effectiveness of such programmes and should be pursued.

FSW intervention spending or coverage may have effects beyond the district in which they operate. Mobility of FSWs and male clients from one district to another is common ${ }^{41}$ and FSW interventions became, by 2008, reasonably uniformly placed across districts (figure 2). ${ }^{14}$ Local FSW interventions might have contributed to a greater public understanding of transmission. Indeed, all high-burden states report marked increases in consistent condom use by FSWs with last client and correct knowledge of HIV transmission. ${ }^{14}$ However, we did not find that district-level changes in self-reported treatment seeking for STI symptoms and correct knowledge of HIV transmission were associated with intervention spending or STIs treated, FSWs reached or condoms distributed or with reductions in risk of HIV or syphilis (data not shown).

Kumar et $a l^{14}$ used the same data from NACO and reported a significant difference in decline in HIV risk in South India among young women attending prenatal clinics from 2001 to 2008 in districts with the highest intervention levels compared with those with the lowest. Their analysis used an indirect exposure measure (condoms distributed to each district), time periods and, importantly, their statistical model did not account for the variation in baseline HIV prevalence, which would have been important as interventions were not randomly assigned. Previous evaluations of the overall impact of Avahan on the risk of HIV in the general population have reported significant effects, particularly in Karnataka. ${ }^{11-13}$ However, our study is the first to compare the Avahan-funded and NACO-funded FSW interventions across the high-burden states. 
FSW interventions and the interruption of STI transmission between FSWs, their male clients, and subsequently to their wives or regular sexual partners remain central to effective control of STI epidemics. FSW interventions need not be costly, and indeed even NACO-funded FSW interventions, which cost about half of the Avahan-funded programmes, were similarly associated with reduced risk of HIV infection and, particularly, reduced risk of syphilis infection.

\section{Author affiliations}

${ }^{1}$ Division of Epidemiology, Centre for Global Health Research, Li Ka Shing Knowledge Institute, St Michael's Hospital, and Dalla Lana School of Public Health, University of Toronto, Toronto, Canada

${ }^{2}$ Department of Community Medicine, UAE University, Al Ain, United Arab Emirates

${ }^{3}$ Department of Family and Community Medicine, Dalla Lana School of Public Health, University of Toronto, Toronto, Canada

${ }^{4}$ Department of Community Health Administration, National Institute of Health and Family Welfare, New Delhi, India

Contributors PA and PJ designed the study in close collaboration with National AIDS Control Organisation (NACO). PA, NJDN, RM and PJ performed the data analysis. All authors contributed to data interpretation and revisions of the manuscript and approved the final version of the manuscript. PA is the guarantor

Funding This study is supported by grants from the Bill and Melinda Gates Foundation (Grant No. 34135), the Canadian Institutes of Health Research (Grant No. 123821) and the University of Toronto (to PJ); and from the Canadian Institutes of Health Research Doctoral Award (to PA). The opinions expressed in this article are those of the authors and do not necessarily represent those of the Government of India, NACO, or the Bill and Melinda Gates Foundation.

Competing interests None.

Provenance and peer review Not commissioned; externally peer reviewed.

Data sharing statement No additional data are available.

\section{REFERENCES}

1. Jha P, Kumar R, Khera A, et al. HIV mortality and infection in India: estimates from nationally representative mortality survey of 1.1 million homes. BMJ 2010;340:c621.

2. Pandey A, Sahu D, Bakkali T, et al. Estimate of HIV prevalence and number of people living with HIV in India 2008-2009. BMJ Open 2012;2:e000926.

3. Kumar R, Jha P, Arora P, et al. HIV-1 trends, risk factors and growth in India. Natl Comm Macroecon Health Background Pap 2005;58-74.

4. Gaffey MF, Venkatesh S, Dhingra N, et al. Male use of female sex work in India: a nationally representative behavioural survey. PLoS ONE 2011;6:e22704.

5. Kumar R, Jha P, Arora P, et al. Trends in HIV-1 in young adults in south India from 2000 to 2004: a prevalence study. Lancet 2006;367:1164-72.

6. Arora $\mathrm{P}, \mathrm{Kumar} \mathrm{R}$, Bhattacharya $\mathrm{M}$, et al. Trends in HIV incidence in India from 2000 to 2007. Lancet 2008;372:289-90.

7. Gangopadhyay DN, Chanda M, Sarkar K, et al. Evaluation of sexually transmitted diseases/human immunodeficiency virus intervention programs for sex workers in Calcutta, India. Sex Transm Dis 2005;32:680-4.

8. National AIDS Control Organization. NACO Annual Report 2011. New Delhi: National AIDS Control Organization, 2011.

9. Claeson M, Alexander A. Tackling HIV in India: evidence-based priority setting and programming. Health Aff 2008;27:1091-102.

10. Mainkar MM, Pardeshi DB, Dale J, et al. Targeted interventions of the Avahan program and their association with intermediate outcomes among female sex workers in Maharashtra, India. BMC Public Health 2011;11:S2.
11. Ng M, Gakidou E, Levin-Rector A, et al. Assessment of population-level effect of Avahan, an HIV-prevention initiative in India. Lancet 2011;378:1643-52.

12. Banandur $\mathrm{P}$, Mahajan U, Potty RS, et al. Population-level impact of Avahan in Karnataka state, south India using multilevel statistical modelling techniques. J Acquir Immune Defic Syndr 2013;62:239-45.

13. Moses S, Bandakoppa MR, Nagelkerke N, et al. Impact of an intensive HIV prevention programme for female sex workers on HIV prevalence among antenatal clinic attenders in Karnataka state, south India: an ecological analysis. AIDS 2008;22(Suppl 5):S101-8.

14. Kumar R, Mehendale SM, Panda S, et al. Impact of targeted interventions on heterosexual transmission of HIV in India. BMC Public Health 2011:11:549.

15. National AIDS Control Organization. National guidelines on prevention, management and control of reproductive tract infections including sexually transmitted infections. New Delhi: Ministry of Health and Family Welfare, 2007

16. International Institute for Population Sciences (IIPS), International M. National Family Health Survey (NFHS-3), 2005-06: India: Volume 1. New Delhi: IIPS Mumbai, India, 2007.

17. National AIDS Control Organization, Health and Family Welfare MO. Annual Report 2009-10. New Delhi, 2010.

18. Hayes R, Watson-Jones D, Celum C, et al. Treatment of sexually transmitted infections for HIV prevention: end of the road or new beginning? AIDS 2010;24:S15.

19. Arora P, Nagelkerke N, Sgaier SK, et al. HIV, HSV-2 and syphilis among married couples in India: patterns of discordance and concordance. Sex Transm Infect 2011;87:516-20.

20. Sgaier SK, Mony P, Jayakumar S, et al. Prevalence and correlates of herpes simplex virus-2 and syphilis infections in the general population in India. Sex Transm Infect 2011;87:94-100.

21. Nandan D, Bhattacharya M. Annual HIV Sentinel Surveillance Country Report 2006. New Delhi: National AIDS Control Organization and National Institute of Health \& Family Welfare.

22. Walker N, Stanecki KA, Brown T, et al. Methods and procedures for estimating HIV/AIDS and its impact: the UNAIDS/WHO estimates for the end of 2001. AIDS 2003;17:2215-25.

23. Group UWW, UNICEF. Epidemiological fact sheet on HIV and AIDS-India. Geneva: UNAIDS, 2008.

24. Mishra S, Moses S, Hanumaiah PK, et al. Sex work, syphilis, and seeking treatment: an opportunity for intervention in HIV prevention programming in Karnataka, South India. Sex Transm Dis 2009;36:157-64.

25. Chesson HW, Pinkerton SD, Voigt R, et al. HIV infections and associated costs attributable to syphilis coinfection among African Americans. Am J Public Health 2003:93:943-8.

26. Reza-Paul S, Beattie T, Syed HUR, et al. Declines in risk behaviour and sexually transmitted infection prevalence following a community-led HIV preventive intervention among female sex workers in Mysore, India. AIDS 2008;22(Suppl 5):S91-100.

27. Watson-Jones D, Weiss HA, Rusizoka M, et al. Effect of herpes simplex suppression on incidence of HIV among women in Tanzania. N Engl J Med 2008;358:1560-71.

28. Celum C, Wald A, Hughes J, et al. Effect of aciclovir on HIV-1 acquisition in herpes simplex virus 2 seropositive women and men who have sex with men: a randomised, double-blind, placebo-controlled trial. Lancet 2008:371:2109-19.

29. Celum C, Wald A, Lingappa JR, et al. Acyclovir and transmission of HIV-1 from persons infected with HIV-1 and HSV-2. N Engl J Med 2010;362:427-39.

30. Kaul R, Kimani J, Nagelkerke NJ, et al. Monthly antibiotic chemoprophylaxis and incidence of sexually transmitted infections and HIV-1 infection in Kenyan sex workers: a randomized controlled trial. JAMA 2004;291:2555-62.

31. Kamali A, Quigley M, Nakiyingi J, et al. Syndromic management of sexually-transmitted infections and behaviour change interventions on transmission of HIV-1 in rural Uganda: a community randomised trial. Lancet 2003;361:645-52.

32. Wawer MJ, Sewankambo NK, Serwadda D, et al. Control of sexually transmitted diseases for AIDS prevention in Uganda: a randomised community trial. Lancet 1999;353:525-35.

33. Rao PJVR. Avahan: the transition to a publicly funded programme as a next stage. Sex Transm Infect 2010;86 (Suppl 1):i7-8.

34. Boily M-C, Pickles M, Vickerman P, et al. Using mathematical modelling to investigate the plausibility of attributing observed antenatal clinic declines to a female sex worker intervention in Karnataka state, India. AIDS 2008;22 (Suppl 5):S149-64.

35. Bhattacharya M, Jaiswal V, Joon V. Supervision and Quality Control Measures in Annual Sentinel Surveillance for HIV Infection in India. $J$ Health Manag 2011;13:329-45. 
36. Registrar General of India. Sample registration system statistical report: 2009. New Delhi: Government of India, 2010.

37. National AIDS Control Organization, National Institute for Medical Statistics. Technical report India HIV estimates 2010. New Delhi: National Institute of Medical Statistics (NIMS); National AIDS Control Organization (NACO), 2012.

38. Ramesh BM, Beattie TSH, Shajy I, et al. Changes in risk behaviours and prevalence of sexually transmitted infections following HIV preventive interventions among female sex workers in five districts in Karnataka state, South India. Sex Transm Infect 2010;86:117-24.

39. Thilakavathi S, Boopathi K, Kumar CG, et al. Assessment of the scale, coverage and outcomes of the Avahan HIV prevention program for female sex workers in Tamil Nadu, India: is there evidence of an effect? BMC Public Health 2011;11:S3

40. Rachakulla HK, Kodavalla V, Rajkumar $\mathrm{H}$, et al. Condom use and prevalence of syphilis and HIV among female sex workers in Andhra Pradesh, India - following a large-scale HIV prevention intervention. BMC Public Health 2011;11:S1.

41. Saggurti N, Verma RK, Jain A, et al. HIV risk behaviours among contracted and non-contracted male migrant workers in India: potential role of labour contractors and contractual systems in HIV prevention. AIDS 2008;22 (Suppl 5): S127-36. 